\title{
TAIL PROPERTIES AND ASYMPTOTIC EXPANSIONS FOR THE MAXIMUM OF THE LOGARITHMIC SKEW-NORMAL DISTRIBUTION
}

\author{
XIN LIAO* AND \\ ZUOXIANG PENG, ${ }^{*}$ Southwest University \\ SARALEES NADARAJAH, ${ }^{* *}$ University of Manchester
}

\begin{abstract}
We discuss tail behaviors, subexponentiality, and the extreme value distribution of logarithmic skew-normal random variables. With optimal normalized constants, the asymptotic expansion of the distribution of the normalized maximum of logarithmic skew-normal random variables is derived. We show that the convergence rate of the distribution of the normalized maximum to the Gumbel extreme value distribution is proportional to $1 /(\log n)^{1 / 2}$.
\end{abstract}

Keywords: Extreme value distribution; logarithmic skew-normal distribution; maximum; pointwise convergence rate; subexponentiality

2010 Mathematics Subject Classification: Primary 62E20; 60G70

Secondary 60F $15 ; 60 \mathrm{~F} 05$

\section{Introduction}

The major weakness of the normal distribution is its inability to model skewed data. Several skewed extensions of the normal distribution have been proposed in the literature. The most popular and the most widely used of these is the skew-normal distribution due to Azzalini (1985). The probability density function (PDF) of this distribution is given by

$$
g_{\lambda}(x)=2 \phi(x) \Phi(\lambda x), \quad x \in \mathbb{R},
$$

where $\lambda \in \mathbb{R}, \phi(x)$ is the standard normal PDF, and $\Phi(x)$ is the standard normal cumulative distribution function (CDF). Let $G_{\lambda}(x)=\int_{-\infty}^{x} g_{\lambda}(t) \mathrm{d} t$ denote the CDF corresponding to (1). If a random variable, say $X$, has $\operatorname{PDF}(1)$ then we write $X \sim \operatorname{SN}(\lambda)$. Clearly, $\operatorname{SN}(0)$ is a standard normal variable.

Liao et al. (2012) studied the tail behavior of the skew-normal distribution, establishing its extreme value distribution and associated convergence rates. The following expansion for the distribution of the normalized maximum of $\mathrm{SN}(\lambda)$ random variables was derived by Liao et al. (2012):

$$
\bar{b}_{n}^{2}\left[\bar{b}_{n}^{2}\left(G_{\lambda}^{n}\left(\bar{a}_{n} x+\bar{b}_{n}\right)-\Lambda(x)\right)-\bar{\kappa}(x) \Lambda(x)\right] \rightarrow\left(\bar{\omega}(x)+\frac{\bar{\kappa}^{2}(x)}{2}\right) \Lambda(x) \quad \text { as } n \rightarrow \infty .
$$

Received 31 August 2012; revision received 6 December 2012.

* Postal address: School of Mathematics and Statistics, Southwest University, 400715 Chongqing, China.

** Postal address: School of Mathematics, University of Manchester, Oxford Road, Manchester, M13 9PL, UK.

Email address: saralees.nadarajah@manchester.ac.uk 
Here $\Lambda(x)=\exp (-\exp (-x))$ denotes the Gumbel CDF and

$$
\begin{aligned}
& \bar{\kappa}(x)=\left(\frac{1}{2} x^{2}+x\right) \mathrm{e}^{-x} \\
& \bar{\omega}(x)=-\left(\frac{1}{8} x^{4}+\frac{1}{2} x^{3}+x^{2}+2 x\right) \mathrm{e}^{-x}
\end{aligned}
$$

with

$$
1-G_{\lambda}\left(\bar{b}_{n}\right)=n^{-1}, \quad \bar{a}_{n}=\bar{b}_{n}^{-1}
$$

for $\lambda \geq 0 ;$ and

$$
\begin{aligned}
& \bar{\kappa}(x)=\left(1+\lambda^{2}\right)^{-1}\left(\frac{1}{2} x^{2}+2 x\right) \mathrm{e}^{-x} \\
& \bar{\omega}(x)=-\lambda^{-2}\left(1+\lambda^{2}\right)^{-2}\left(\frac{1}{8} \lambda^{2} x^{4}+\lambda^{2} x^{3}+3 \lambda^{2} x^{2}+2\left(1+3 \lambda^{2}\right) x\right) \mathrm{e}^{-x}
\end{aligned}
$$

with

$$
1-G_{\lambda}\left(\bar{b}_{n}\right)=n^{-1}, \quad \bar{a}_{n}=\left(\left(1+\lambda^{2}\right) \bar{b}_{n}\right)^{-1}
$$

for $\lambda<0$.

The skew-normal distribution applies to data on the real line. Its version for positive data can be obtained by setting $X=\exp (\xi)$, where $\xi \sim \mathrm{SN}(\lambda)$. Then we say that $X$ follows the logarithmic skew-normal distribution, written as $X \sim \operatorname{LSN}(\lambda)$. The PDF of $\operatorname{LSN}(\lambda)$ is given by

$$
f_{\lambda}(x)=\frac{2}{x} \phi(\log x) \Phi(\lambda \log x), \quad x>0 .
$$

Let $F_{\lambda}(\cdot)$ denote the CDF corresponding to (2). Clearly, $\mathrm{LSN}(0)$ is a standard log-normal random variable.

The logarithmic skew-normal distribution is more recent than the skew-normal distribution, but it has already led to widespread applications. Some selected applications and application areas have been modeling of income data (see Azzilini et al. (2003)); analysis of auto insurance claim costs (see Bolance et al. (2008)); analysis of continuous data in a two-part stochastic model (see Chai and Bailey (2008)); wireless communications (see Wu et al. (2009) and Li et al. (2011)); modeling of particle size (see Huang and Ku (2010)); cohort studies of paediatric respiratory symptoms (see Mahmud et al. (2010)); and modeling of precipitation data (see Marchenko and Genton (2010)). Some probabilistic properties of $\operatorname{LSN}(\lambda)$ have been studied in Lin and Stoyanov (2009).

The aim of this short note is to consider some further probabilistic properties of the logarithmic skew-normal distribution. The contents are organized as follows. In Section 2 we present some preliminary results, including the tail behavior, the subexponentiality, and the extreme value distribution of $\operatorname{LSN}(\lambda)$. Distributional expansions for the normalized maximum of $\operatorname{LSN}(\lambda)$ random variables are derived in Section 3. To the best of our knowledge, all of the properties presented are new.

\section{Preliminary results}

In this section we derive Mills' inequalities, Mills' ratios, and an exact decomposition of the tail of $\operatorname{LSN}(\lambda)$. We also prove that $\operatorname{LSN}(\lambda)$ is strongly subexponential, denoted by $F_{\lambda} \in 8^{*}$.

For $\operatorname{LSN}(\lambda)$ and $\operatorname{SN}(\lambda)$, note that $1-F_{\lambda}(x)=1-G_{\lambda}(\log x)$ and

$$
\frac{1-F_{\lambda}(x)}{f_{\lambda}(x)}=x \frac{1-G_{\lambda}(\log x)}{g_{\lambda}(\log x)} .
$$


So, by Proposition 1 of Liao et al. (2012) and by Mills' inequality and Mills' ratio of the standard normal distribution, we have the following two results.

Proposition 1. Let $F_{\lambda}(x)$ and $f_{\lambda}(x)$ denote the CDF and the PDF of $\operatorname{LSN}(\lambda)$. For all $x>1$,

(i) if $\lambda>0$,

$$
\frac{x}{\log x}\left(1+(\log x)^{-2}\right)^{-1}<\frac{1-F_{\lambda}(x)}{f_{\lambda}(x)}<\frac{x}{\log x}\left(1-\frac{\phi(\lambda \log x)}{\lambda \log x}\right)^{-1}
$$

(ii) if $\lambda=0$,

$$
\frac{x}{\log x}\left(1+(\log x)^{-2}\right)^{-1}<\frac{1-F_{0}(x)}{f_{0}(x)}<\frac{x}{\log x}
$$

(iii) if $\lambda<0$,

$$
\begin{aligned}
\frac{x}{\log x} & \left(1+(\log x)^{-2}\right)^{-1}\left(1-\frac{\lambda^{2}}{1+\lambda^{2}}\left(1+\frac{1}{\lambda^{2}(\log x)^{2}}\right)\right) \\
& <\frac{1-F_{\lambda}(x)}{f_{\lambda}(x)}<\frac{x}{\log x}\left(1-\frac{\lambda^{2}}{1+\lambda^{2}}\left(1+\frac{1}{\left(1+\lambda^{2}\right)(\log x)^{2}}\right)^{-1}\right) .
\end{aligned}
$$

Proposition 2. Let $F_{\lambda}(x)$ and $f_{\lambda}(x)$ denote the CDF and the PDF of $\operatorname{LSN}(\lambda)$. For $\lambda \geq 0$, we have

$$
\frac{1-F_{\lambda}(x)}{f_{\lambda}(x)} \sim \frac{x}{\log x} \text { as } x \rightarrow \infty
$$

For $\lambda<0$, we have

$$
\frac{1-F_{\lambda}(x)}{f_{\lambda}(x)} \sim \frac{x}{\left(1+\lambda^{2}\right) \log x} \text { as } x \rightarrow \infty .
$$

The following result shows that $\operatorname{LSN}(\lambda)$ is strongly subexponential.

Corollary 1. $F_{\lambda} \in \mathcal{S}^{*}$, so $F_{\lambda} \in \&$, the class of subexponential distributions.

Proof. By Proposition 2, the hazard rate function $m_{F_{\lambda}}(x)=f_{\lambda}(x) /\left(1-F_{\lambda}(x)\right)$ is ultimately decreasing to 0 as $x \rightarrow \infty$. If $\exp \left(x m_{F_{\lambda}}(x)\right) \bar{F}_{\lambda}(x)$ is integrable over $\mathbb{R}^{+}$, where $\bar{F}_{\lambda}(x)=$ $1-F_{\lambda}(x)$, Theorem 3.32 of Foss et al. (2011) shows that $F_{\lambda} \in 8^{*}$. Combining this with Theorem 3.27 of Foss et al. (2011), we have $F_{\lambda} \in 8$. So, we just need to check that $\exp \left(x m_{F_{\lambda}}(x)\right) \bar{F}_{\lambda}(x)$ is integrable over $\mathbb{R}^{+}$.

Consider the $\lambda \geq 0$ case. By (3) we know for arbitrary $\varepsilon>0$ that there exists a sufficiently large $A>0$ such that

$$
(1-\varepsilon) \frac{x}{\log x}<\frac{1-F_{\lambda}(x)}{f_{\lambda}(x)}<(1+\varepsilon) \frac{x}{\log x} .
$$

Hence, for $x>A$, we have

$$
\begin{aligned}
\exp \left(x m_{F_{\lambda}}(x)\right) \bar{F}_{\lambda}(x) & <(1+\varepsilon) \frac{x f_{\lambda}(x)}{\log x} \exp \left(\frac{1}{1-\varepsilon} \log x\right) \\
& <\frac{2(1+\varepsilon)}{\log A} \phi(\log x) \exp \left(\frac{1}{1-\varepsilon} \log x\right) \\
& =\frac{2(1+\varepsilon)}{\log A} \exp \left(\frac{1}{2(1-\varepsilon)^{2}}\right) \phi\left(\log x-\frac{1}{1-\varepsilon}\right) .
\end{aligned}
$$


So, we can check that $\lim _{x \rightarrow \infty} x^{k} \exp \left(x m_{F_{\lambda}}(x)\right) \bar{F}_{\lambda}(x)=0$ for any $k>1$, implying that $\exp \left(x m_{F_{\lambda}}(x)\right) \bar{F}_{\lambda}(x)$ is integrable over $\mathbb{R}^{+}$.

The same can be shown for the $\lambda<0$ case by using (4). The arguments are similar and thus omitted. This completes the proof.

In order to derive expansions for the distribution of the normalized maximum of $\operatorname{LSN}(\lambda)$ random variables, we need the following tail decomposition of $\operatorname{LSN}(\lambda)$.

Proposition 3. Let $F_{\lambda}(x)$ denote the $C D F$ of $\operatorname{LSN}(\lambda)$. Then, for large $x$, if $\lambda \geq 0$, we have

$$
\begin{aligned}
1-F_{\lambda}(x)= & \frac{f_{\lambda}(\log x)}{\log x}\left(1-(\log x)^{-2}+3(\log x)^{-4}+O\left((\log x)^{-6}\right)\right) \\
= & \sqrt{\frac{2}{\pi \mathrm{e}}} \Phi(\lambda \log x)\left(1-(\log x)^{-2}+3(\log x)^{-4}+O\left((\log x)^{-6}\right)\right) \\
& \times \exp \left(-\int_{\mathrm{e}}^{x} \frac{\log s}{s}\left(1+(\log s)^{-2}\right) \mathrm{d} s\right) .
\end{aligned}
$$

If $\lambda<0$, we have

$$
\begin{aligned}
1-F_{\lambda}(x)= & \frac{\exp \left(-\left(1+\lambda^{2}\right)(\log x)^{2} / 2\right)}{(-\lambda) \pi\left(1+\lambda^{2}\right)(\log x)^{2}} \\
& \times\left(1-\frac{1+3 \lambda^{2}}{\lambda^{2}\left(1+\lambda^{2}\right)}(\log x)^{-2}+\frac{15 \lambda^{4}+10 \lambda^{2}+3}{\lambda^{4}\left(1+\lambda^{2}\right)^{2}}(\log x)^{-4}+O\left((\log x)^{-6}\right)\right) \\
= & \frac{\exp \left(-\left(1+\lambda^{2}\right) / 2\right)}{(-\lambda) \pi\left(1+\lambda^{2}\right)} \\
& \times\left(1-\frac{1+3 \lambda^{2}}{\lambda^{2}\left(1+\lambda^{2}\right)}(\log x)^{-2}+\frac{15 \lambda^{4}+10 \lambda^{2}+3}{\lambda^{4}\left(1+\lambda^{2}\right)^{2}}(\log x)^{-4} O\left((\log x)^{-6}\right)\right) \\
& \times \exp \left(-\int_{\mathrm{e}}^{x} \frac{\left(1+\lambda^{2}\right) \log s}{s}\left(1+\frac{2}{\left(1+\lambda^{2}\right)(\log s)^{2}}\right) \mathrm{d} s\right) .
\end{aligned}
$$

Proof. The proof follows by integration by parts.

Using Proposition 3, we can now derive the distributional tail representation of $\operatorname{LSN}(\lambda)$.

Proposition 4. For large $x$,

$$
1-F_{\lambda}(x)=c(x) \exp \left(-\int_{\mathrm{e}}^{x} \frac{g(t)}{f(t)} \mathrm{d} t\right),
$$

where $c(x), g(x)$, and $f(x)$ depend on $\lambda$ as follows. In the $\lambda \geq 0$ case,

$$
\begin{gathered}
c(x) \rightarrow \sqrt{\frac{2}{\pi \mathrm{e}}} \text { as } x \rightarrow \infty, \\
f(x)=\frac{x}{\log x}>0 \quad \text { with } f^{\prime}(x)=-\frac{\log x-1}{(\log x)^{2}} \rightarrow 0 \quad \text { as } x \rightarrow \infty,
\end{gathered}
$$

and

$$
g(x)=1+\frac{1}{(\log x)^{2}} \rightarrow 1 \quad \text { as } x \rightarrow \infty
$$


In the $\lambda<0$ case,

$$
\begin{gathered}
c(x) \rightarrow \frac{\exp \left(-\left(1+\lambda^{2}\right) / 2\right)}{(-\lambda) \pi\left(1+\lambda^{2}\right)} \text { as } x \rightarrow \infty, \\
f(x)=\frac{x}{\left(1+\lambda^{2}\right) \log x}>0 \quad \text { with } f^{\prime}(x)=-\frac{\log x-1}{\left(1+\lambda^{2}\right)(\log x)^{2}} \rightarrow 0 \quad \text { as } x \rightarrow \infty,
\end{gathered}
$$

and

$$
g(x)=1+\frac{2}{\left(1+\lambda^{2}\right)(\log x)^{2}} \rightarrow 1 \quad \text { as } x \rightarrow \infty .
$$

In fact, Proposition 4 can also be obtained from Mills' ratio of $\operatorname{LSN}(\lambda)$. By Corollary 1.7 of Resnick (1987), we have $F_{\lambda} \in D(\Lambda)$, and the norming constants $a_{n}$ and $b_{n}$ are given by

$$
n^{-1}=1-F_{\lambda}\left(b_{n}\right), \quad a_{n}=f\left(b_{n}\right)
$$

such that

$$
\lim _{n \rightarrow \infty} F_{\lambda}^{n}\left(a_{n} x+b_{n}\right)=\Lambda(x) .
$$

Remark 1. The tail representation of $\operatorname{LSN}(\lambda)$ can be rewritten as

$$
1-F_{\lambda}(x)=c(x) \exp \left(-\int_{\mathrm{e}}^{x} \frac{1}{f^{*}(t)} \mathrm{d} t\right)
$$

with $f^{*}(x)=f(t) / g(t)$ eventually nondecreasing, where $c(x), f(t)$, and $g(t)$ are those given in Proposition 4. By Corollary 2.5 of Goldie and Resnick (1988), we can easily check that $F_{\lambda} \in \delta \cap D(\Lambda)$ since $\lim _{x \rightarrow \infty} f^{*}(h x) / f^{*}(x)=h$ for any constant $h>1$.

\section{Expansion for the distribution of the maximum}

In this section we derive an exact expansion for the distribution of the maximum of $\operatorname{LSN}(\lambda)$ random variables. This expansion is used to show that the convergence rate of $F_{\lambda}^{n}\left(a_{n} x+b_{n}\right)$ to $\Lambda(x)$ is of the order of $O\left((\log n)^{-1 / 2}\right)$.

Theorem 1. For norming constants $a_{n}$ and $b_{n}$ given in (6), we have

$$
\left(\log b_{n}\right)\left(\left(\log b_{n}\right)\left(F_{\lambda}^{n}\left(a_{n} x+b_{n}\right)-\Lambda(x)\right)-\kappa(x) \Lambda(x)\right) \rightarrow\left(\omega(x)+\frac{\kappa^{2}(x)}{2}\right) \Lambda(x)
$$

as $n \rightarrow \infty$, where $\kappa(x)$ and $\omega(x)$ depend on $\lambda$ as follows. In the $\lambda \geq 0$ case,

$$
\begin{aligned}
& \kappa(x)=-2^{-1} x^{2} \mathrm{e}^{-x}, \\
& \omega(x)=-24^{-1}\left(3 x^{4}-8 x^{3}-12 x^{2}-24 x\right) \mathrm{e}^{-x}
\end{aligned}
$$

in the $\lambda<0$ case,

$$
\begin{aligned}
& \kappa(x)=-2^{-1}\left(1+\lambda^{2}\right)^{-1} x^{2} \mathrm{e}^{-x} \\
& \omega(x)=-24^{-1}\left(1+\lambda^{2}\right)^{-2}\left(3 x^{4}-8 x^{3}-12\left(1+\lambda^{2}\right) x^{2}-48\left(1+\lambda^{2}\right) x\right) \mathrm{e}^{-x} .
\end{aligned}
$$

To prove Theorem 1, we need the following auxiliary result. 
Lemma 1. Let $H_{\lambda}\left(b_{n} ; x\right)=F_{\lambda}\left(a_{n} x+b_{n}\right)$ and $h_{\lambda}\left(b_{n} ; x\right)=n \log H_{\lambda}\left(b_{n} ; x\right)+\mathrm{e}^{-x}$, where the norming constants $a_{n}$ and $b_{n}$ are given in (6). Then

$$
\lim _{n \rightarrow \infty}\left(\log b_{n}\right)\left(\left(\log b_{n}\right) h_{\lambda}\left(b_{n} ; x\right)-\kappa(x)\right)=\omega(x),
$$

where $\kappa(x)$ and $\omega(x)$ are those given in Theorem 1 .

Proof. First, consider the $\lambda \geq 0$ case. It is easy to check the following two facts by (3) and $F_{\lambda} \in D(\Lambda)$ :

$$
\lim _{n \rightarrow \infty} n\left(1-F_{\lambda}\left(\frac{b_{n}}{\log b_{n}} x+b_{n}\right)\right)=\mathrm{e}^{-x}
$$

and

$$
\lim _{n \rightarrow \infty}\left(1-F_{\lambda}\left(\frac{b_{n}}{\log b_{n}} x+b_{n}\right)\right)\left(\log b_{n}\right)^{2}=0 .
$$

Setting

$$
\begin{aligned}
A_{\lambda}\left(b_{n}\right)=[ & \left.\Phi\left(\lambda \log b_{n}\right)\left(1-\left(\log b_{n}\right)^{-2}+3\left(\log b_{n}\right)^{-4}+O\left(\left(\log b_{n}\right)^{-6}\right)\right)\right] \\
\times & {\left[3\left(\log \left(\frac{b_{n}}{\log b_{n}} x+b_{n}\right)\right)^{-4}\right.} \\
& +\Phi\left(\lambda \log \left(\frac{b_{n}}{\log b_{n}} x+b_{n}\right)\right) \\
& \left.\times\left(1-\left(\log \left(\frac{b_{n}}{\log b_{n}} x+b_{n}\right)\right)^{-2}+O\left(\left(\log b_{n}\right)^{-6}\right)\right)\right]^{-1},
\end{aligned}
$$

we have $\lim _{n \rightarrow \infty} A_{\lambda}\left(b_{n}\right)=1$ and

$$
\lim _{n \rightarrow \infty}\left(A_{\lambda}\left(b_{n}\right)-1\right)\left(\log b_{n}\right)^{2}=0 .
$$

So, by (5), we have

$$
\begin{gathered}
\frac{1-F_{\lambda}\left(b_{n}\right)}{1-F_{\lambda}\left(b_{n} x / \log b_{n}+b_{n}\right)} \mathrm{e}^{-x} \\
=A_{\lambda}\left(b_{n}\right) \exp \left(\int_{b_{n}}^{b_{n}+b_{n} x / \log b_{n}} \frac{\log s}{s}\left(1+\frac{1}{(\log s)^{2}}\right) \mathrm{d} s-x\right) \\
=A_{\lambda}\left(b_{n}\right) \exp \left(\int _ { 0 } ^ { x } \left(\frac{-t+\log \left(1+t / \log b_{n}\right)}{\log b_{n}+t}\right.\right. \\
\left.\left.\quad+\frac{1}{\left(\log b_{n}+t\right)\left(\log b_{n}+\log \left(1+t / \log b_{n}\right)\right)}\right) \mathrm{d} t\right) \\
=A_{\lambda}\left(b_{n}\right)\left(1+\int_{0}^{x}\left(\frac{-t+\log \left(1+t / \log b_{n}\right)}{\log b_{n}+t}\right) \mathrm{d} t\right. \\
+\frac{1+o(1)}{2}\left(\int _ { 0 } ^ { x } \left(\frac{-t+\log \left(1+t / \log b_{n}\right)}{\log b_{n}+t}\right.\right. \\
\left.\left.\left.+\frac{1}{\left(\log b_{n}+t\right)\left(\log b_{n}+\log \left(1+t / \log b_{n}\right)\right)}\right) \mathrm{d} t\right)^{2}\right) .
\end{gathered}
$$


Combining (3), (7), (8), (9), and (10), we obtain

$$
\begin{aligned}
& \lim _{n \rightarrow \infty}\left(\log b_{n}\right) h_{\lambda}\left(b_{n} ; x\right) \\
& =\lim _{n \rightarrow \infty} \frac{n \log H_{\lambda}\left(b_{n} ; x\right)+\mathrm{e}^{-x}}{\left(\log b_{n}\right)^{-1}} \\
& =\lim _{n \rightarrow \infty} \frac{n\left(\log F_{\lambda}\left(b_{n} x / \log b_{n}+b_{n}\right)+\left(1-F_{\lambda}\left(b_{n}\right)\right) \mathrm{e}^{-x}\right)}{\left(\log b_{n}\right)^{-1}} \\
& =\lim _{n \rightarrow \infty} \frac{n\left(-\left(1-F_{\lambda}\left(b_{n} x / \log b_{n}+b_{n}\right)\right)-(1 / 2)\left(1-F_{\lambda}\left(b_{n} x / \log b_{n}+b_{n}\right)\right)^{2}(1+o(1))\right)}{\left(\log b_{n}\right)^{-1}} \\
& +\lim _{n \rightarrow \infty} \frac{n\left(1-F_{\lambda}\left(b_{n}\right)\right) \mathrm{e}^{-x}}{\left(\log b_{n}\right)^{-1}} \\
& =\lim _{n \rightarrow \infty} \frac{n\left(1-F_{\lambda}\left(b_{n} x / \log b_{n}+b_{n}\right)\right)\left(-1-(1 / 2)\left(1-F_{\lambda}\left(b_{n} x / \log b_{n}+b_{n}\right)\right)(1+o(1))\right)}{\left(\log b_{n}\right)^{-1}} \\
& +\lim _{n \rightarrow \infty} \frac{n\left(1-F_{\lambda}\left(b_{n} x / \log b_{n}+b_{n}\right)\right)\left(1-F_{\lambda}\left(b_{n}\right)\right) \mathrm{e}^{-x} /\left(1-F_{\lambda}\left(b_{n} x / \log b_{n}+b_{n}\right)\right)}{\left(\log b_{n}\right)^{-1}} \\
& =\mathrm{e}^{-x} \lim _{n \rightarrow \infty} \frac{-1+\left(1-F_{\lambda}\left(b_{n}\right)\right) \mathrm{e}^{-x} /\left(1-F_{\lambda}\left(b_{n} x / \log b_{n}+b_{n}\right)\right)}{\left(\log b_{n}\right)^{-1}} \\
& =\mathrm{e}^{-x} \lim _{n \rightarrow \infty} \frac{-1+A_{\lambda}\left(b_{n}\right)\left(1+\int_{0}^{x}\left(-t+\log \left(1+t / \log b_{n}\right)\right)\left(\log b_{n}+t\right)^{-1} \mathrm{~d} t(1+o(1))\right)}{\left(\log b_{n}\right)^{-1}} \\
& +\mathrm{e}^{-x} \lim _{n \rightarrow \infty} \frac{A_{\lambda}\left(b_{n}\right) \int_{0}^{x}\left(\left(\log b_{n}+t\right)\left(\log b_{n}+\log \left(1+t / \log b_{n}\right)\right)\right)^{-1} \mathrm{~d} t(1+o(1))}{\left(\log b_{n}\right)^{-1}} \\
& =\mathrm{e}^{-x} \lim _{n \rightarrow \infty} \frac{A_{\lambda}\left(b_{n}\right)-1+A_{\lambda}\left(b_{n}\right) \int_{0}^{x}\left(-t+\log \left(1+t / \log b_{n}\right)\right)\left(\log b_{n}+t\right)^{-1} \mathrm{~d} t(1+o(1))}{\left(\log b_{n}\right)^{-1}} \\
& +\mathrm{e}^{-x} \lim _{n \rightarrow \infty} \frac{A_{\lambda}\left(b_{n}\right) \int_{0}^{x}\left(\left(\log b_{n}+t\right)\left(\log b_{n}+\log \left(1+t / \log b_{n}\right)\right)\right)^{-1} \mathrm{~d} t(1+o(1))}{\left(\log b_{n}\right)^{-1}} \\
& =\mathrm{e}^{-x} \lim _{n \rightarrow \infty} \int_{0}^{x}\left(\frac{-t+\log \left(1+t / \log b_{n}\right)}{1+t / \log b_{n}}\right. \\
& \left.+\frac{1}{\left(1+t / \log b_{n}\right)\left(\log b_{n}+\log \left(1+t / \log b_{n}\right)\right)}\right) \mathrm{d} t \\
& =-\frac{1}{2} x^{2} \mathrm{e}^{-x} \\
& :=\kappa(x) \text {, }
\end{aligned}
$$

where the final step follows by the dominated convergence theorem. Similarly, we can show that $\lim _{n \rightarrow \infty}\left(\log b_{n}\right)\left(\left(\log b_{n}\right) h_{\lambda}\left(b_{n} ; x\right)-\kappa(x)\right)=\omega(x)$.

The same results hold for $\lambda>0$ by (4) and Proposition 4. The arguments are similar and thus omitted. This completes the proof.

Proof of Theorem 1. Note that $\lim _{n \rightarrow \infty} h_{\lambda}\left(b_{n} ; x\right)=0$ by Lemma 1. Using Lemma 1 again, we have

$$
\begin{aligned}
& \left(\log b_{n}\right)\left(\left(\log b_{n}\right)\left(F_{\lambda}\left(a_{n} x+b_{n}\right)-\Lambda(x)\right)-\kappa(x) \Lambda(x)\right) \\
& \quad=\left(\log b_{n}\right)\left(\left(\log b_{n}\right)\left(\exp \left(h_{\lambda}\left(b_{n} ; x\right)-1\right)\right)-\kappa(x)\right) \Lambda(x) \\
& \quad=\left(\log b_{n}\right)\left(\left(\log b_{n}\right)\left(h_{\lambda}\left(b_{n} ; x\right)+\frac{h_{\lambda}^{2}\left(b_{n} ; x\right)}{2}+\frac{h_{\lambda}^{3}\left(b_{n} ; x\right)}{3 !}(1+o(1))\right)-\kappa(x)\right)
\end{aligned}
$$




$$
\begin{aligned}
& =\left(\left(\log b_{n}\right)\left(\left(\log b_{n}\right) h_{\lambda}\left(b_{n} ; x\right)-\kappa(x)\right)\right. \\
& \left.\quad+\left(\log b_{n}\right)^{2} h_{\lambda}^{2}\left(b_{n} ; x\right)\left(\frac{1}{2}+\frac{h_{\lambda}\left(b_{n} ; x\right)}{3 !}(1+o(1))\right)\right) \Lambda(x) \\
& \rightarrow\left(\omega(x)+\frac{\kappa^{2}(x)}{2}\right) \Lambda(x) \text { as } n \rightarrow \infty
\end{aligned}
$$

The desired result follows.

Remark 2. By the definition of $b_{n}$, it is easy to check that $1 / \log b_{n}=O\left(1 /(\log n)^{1 / 2}\right)$. So, Theorem 1 shows that the pointwise convergence rate of $F_{\lambda}^{n}\left(a_{n} x+b_{n}\right)$ to its limit is proportional to $1 /(\log n)^{1 / 2}$. Furthermore, the pointwise convergence rate of $\left(\log b_{n}\right)\left(F_{\lambda}^{n}\left(a_{n} x+b_{n}\right)-\Lambda(x)\right)$ to its limit is also proportional to $1 /(\log n)^{1 / 2}$ by Theorem 1 .

\section{Acknowledgements}

The first two authors' research was supported by the National Science Foundation of China under grant 11171275 and an SWU grant for a statistics PhD program. All of the authors would like to thank the Editor and the referee for careful readings and comments which greatly improved the paper.

\section{References}

Azzalini, A. (1985). A class of distributions which includes the normal ones. Scand. J. Statist. 12, 171-178.

Azzalini, A., Dei Cappello, T. And Kotz, S. (2003). Log-skew-normal and log-skew-t distributions as models for family income data. J. Income Distribution 11, 12-20.

Bolance, C., Guillen, M., Pelican, E. and Vernic, R. (2008). Skewed bivariate models and nonparametric estimation for the CTE risk measure. Insurance Math. Econom. 43, 386-393.

ChaI, H. S. AND BAILey, K. R. (2008). Use of log-skew-normal distribution in analysis of continuous data with a discrete component at zero. Statist. Medicine 27, 3643-3655.

Foss, S., Korshunov, D. And Zachary, S. (2011). An Introduction to Heavy-Tailed and Subexponential Distributions. Springer, New York.

Goldie, C. M. AND Resnick, S. (1988). Distributions that are both subexponential and in the domain of attraction of an extreme-value distribution. J. Appl. Prob. 20, 706-718.

Huang, C. Y. AND Ku, M. S. (2010). Asymmetry effect of particle size distribution on content uniformity and overpotency risk in low-dose solid drugs. J. Pharm. Sci. 99, 4351-4362.

Li, X., Wu, Z., Chakravarthy, V. D. and Wu, Z. (2011). A low-complexity approximation to lognormal sum distributions via transformed log skew normal distribution. IEEE Trans. Vehicular Tech. 60, 4040-4045.

Liao, X., Peng, Z., Nadarajah, S. and Wang, X. (2012). Rates of convergence of extremes from skew normal samples. Preprint. Available at http://arxiv.org/abs/1212.1004v1.

Lin, D. G. And Stoyanov, J. (2009). The logarithmic skew-normal distributions are moment-indeterminate. J. Appl. Prob. 46, 909-916.

Mahmud, S., Lou, W. Y. W. And Johnston, N. W. (2010). A probit- log- skew-normal mixture model for repeated measures data with excess zeros, with application to a cohort study of paediatric respiratory symptoms. $B M C$ Medical Res. Methodology 10, article no. 55.

Marchenko, Y. V. And Genton, M. G. (2010). Multivariate log-skew-elliptical distributions with applications to precipitation data. Environmetrics 21, 318-340.

ReSnick, S. I. (1987). Extreme Values, Regular Variation, and Point Processes. Springer, New York.

Wu, Z. et al. (2009). A novel highly accurate log skew normal approximation method to lognormal sum distributions. In Proc. 2009 IEEE Wireless Communications and Networking Conf., IEEE pp. 392-397. 